\title{
The Wing Pavilion:A Participatory Action Approach to Bamboo Architectural Research in Chinese Universities
}

\author{
Daniel Stamatis* Wang Zhen Liu Hui Tan Gangyi \\ School of Architecture and Urban Planning, Huazhong University of Science and Technology, 1037
}

\begin{abstract}
The application of bamboo construction techniques in Chinese architecture remains behind its potential. Chinese universities began conducting participatory action research on bamboo as a building material. Students from the Huazhong University of Science and Technology designed the wing pavilion: a temporary bamboo structure built in six days inside the Nan Sha Bird Eco-Park, Guangzhou. The process combined handcraft and digital techniques within five steps: pre-design, formulation, successive answers, project, and conclusion of the theory. Eight bamboo arches interconnected to create a six-meter span roof. Each arch was pre-fabricated with two layers of split bamboo and one slender bamboo culm as reinforcement. The cartesian plane technique was utilised to overcome the indeterminacy of bamboo. Details could be fuller, and the roof lacked enough strength. More important than a perfect result, the principles of bamboo architecture transferred to students, and a range of scales can replicate the methodology. The technical solution reflected a Western functionality with an Asian essence. Many of the now underprivileged people will benefit from it since bamboo is abundant in China.
\end{abstract}

Keywords: Bamboo architecture, participatory action research, green building, architectural education.

DOI: $10.7176 / \mathrm{ISDE} / 10-5-03$

Publication date:June $30^{\text {th }} 2019$

\section{Introduction}

Bamboo: fast growth, high adaptability, substantial biomass production, with the tensile strength higher than mild steel, and able to resist compression efforts twice as well as concrete, is probably the most multi-use natural product available (Van der Lugt, 2017). In China, bamboo is in our daily-life since ancient times. Nowadays, the Chinese bamboo industry is valued at 30 billion USD, offering a large variety of products: from the more traditional such as baskets, mats, or as food, to the more sophisticated such as wind turbine blades, shock-resistant exteriors for bullet-train carriages, or storm-drainage pipes (Friederich, 2019). In many cases, bamboo is a possible substitute for plastic, wood, or steel. However, compared to the industry's growth, the application of bamboo construction techniques in Chinese architecture remains behind its potential. Generally, most of the bamboo is applied as a wood, overlooking its excellent mechanical properties (Xinping et al. 2018). Even though research on bamboo has been dominated mainly by the agronomy and forestry sectors, the increasing demand for sustainable construction has been encouraging the areas of architecture and design to conduct more research on bamboo as a building material (Marsden et al., 2011). However, research on bamboo as a building material is not new. For instance, in 1952, McClure had already published a comprehensive report showing most of the features utilised even today. Since then, the joinery systems have made little progress. Researchers abandoned the subject due to the focus on industrialised materials such as steel, concrete, and timber (McClure, 1982).

Although the bamboo's adaptability to human needs has few peers in the plant kingdom, its utilisation in architecture still under its potential. INBAR (The International Network for Bamboo and Rattan) recognises that a critical obstacle is the lack of information among the general population, especially in the rural dwellers and policymakers (INBAR, 2018). The solution to this lack of awareness relies mainly on the provision of training to people in different sectors. For instance, the Ministry of Commerce of the People's Republic of China with the CBRC China National Bamboo Research Center, based in Hangzhou, have been providing training on bamboo cultivation, processing and utilisation for Asia, South America and Africa. Nonetheless, most of these training does not focus enough on as a building material. For the sector of architecture, Chinese universities play a critical role in the popularisation of bamboo as a building material. The consciousness of environmental issues and the presence of digital design techniques among younger architects are accelerating the process (Crolla, 2017). Recent studies show that a bamboo architectural practice helps to reduce the gap between the aspirations of modernity of rural dwellers and the ethnic reinforcement. The rural-to-urban migration process is related to the subject. The bamboo resource is abundant in China (Botz-Bornstein, 2009; Flynn et al., 2017; Wang, 2012). This article reports the process and results of the participatory action research on bamboo architecture conducted in 2018 by architecture students at Huazhong University of Science and Technology. Domestic and international bamboo experts evaluated the learnability and innovation of the project's construction techniques with quantitative and qualitative approaches. 
more than 20 universities, two cases are presented as a non-exhaustive analysis to illustrate the critical principles that influenced the process of decision-making of the wing pavilion: 1) the cocoon duck shelter's case, by the Southeast University, and 2) the bamboo bridge truss' case by the University of Hong Kong. This section will conclude with a brief description of the call-to-action from the Nan Sha Bird Eco-Park of Guangzhou, who invited the students from the Huazhong University of Science and Technology to have a real experience on what it takes to build with bamboo.

\subsubsection{The 'cocoon' duck shelter, by Southeast University}

In 2015, Professor Zhang Tong from Southeast University guided 32 graduate students to conduct participatory action research on natural bamboo and low-tech construction in the Shuangmiao Village, Zhejiang Province. The goal was to build 22 duck shelters due to the local production mode of "rice-duck farming" requiring strict affiliation between ducks and rice fields. The "cocoon" was one of those shelters, designed by Zhong Wenzhou, Ma Danhong, and Zheng Xing. During five months, the students researched the field, investigated about bamboo as a building material, generated the design concept, defined the specific joinery, and finally built it on-site. During the research phase, bamboo was discovered as a sturdy material but with a different characteristic to it: an extraordinary capacity of bending and weaving. If "poetic construction" is discussed in terms of how to use bamboo in its way, then bending and weaving is undoubtedly the most suitable for bamboo material. The design aimed to approach the construction as a natural and primitive "home". A simple piece of bamboo bent into an arch, and the lower members were connected to form the space of a duck shelter. Craftsmanship relied on the long-term experience of the local workforce. The three students had no bamboo work experience. Therefore, the design strived to reduce the need for construction accuracy, and choose to "scramble" this weaving method to ensure the completion effect and allow errors within a range. The final construction logic was such that the split bamboo arches formed the basic structure's shape and then reinforced it by irregular weaving to create a nest-like natural texture (Figure 1).
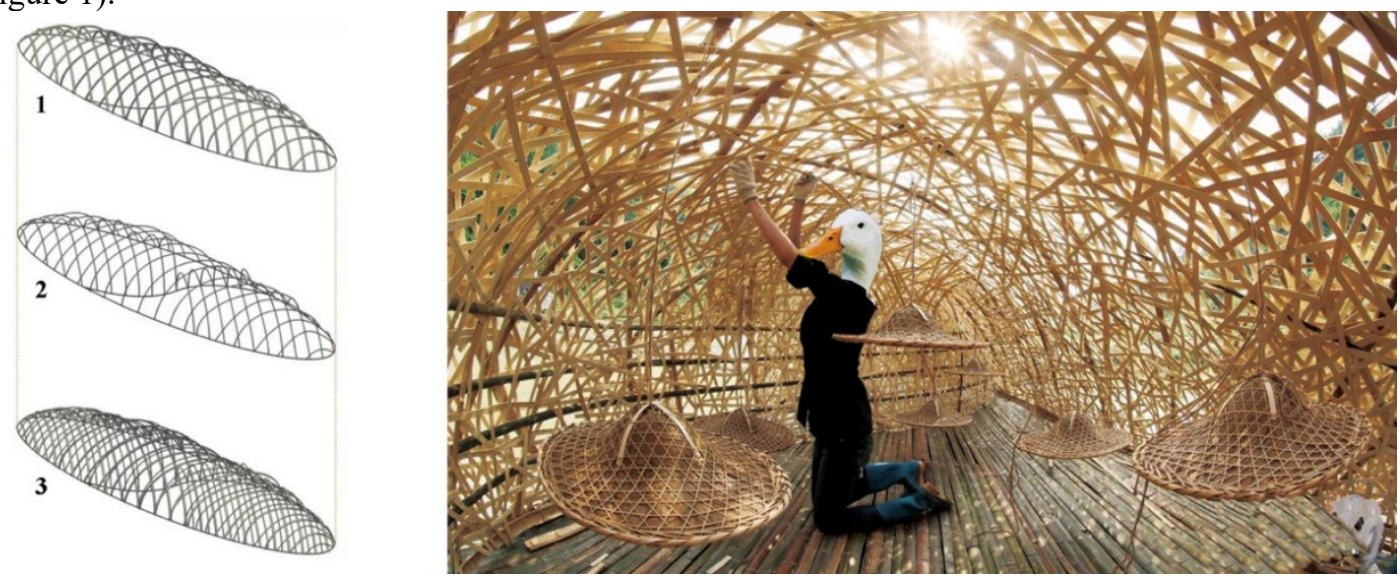

Figure 1. Weaving bamboo in the interior of the shelter after three layers of split bamboo arches. Created by Zhong Wenzhou, Ma Danhong, and Zheng Xing.

The volume was a nearly $12 \mathrm{~m}$ length and $3.2 \mathrm{~m}$ width. It was built in the village and transported to the site as a unit using a small truck. The original design proposed to use lashing for the connection between the arches and the bottom surface. However, such an operation was too slow. Therefore local craftsman suggested using gun nails instead of lashing, which was more comfortable and faster to operate. The gun-mail node did not reflect the construction characteristics of bamboo, but the low-efficiency of lashing node was contrary to the constructive rationality. These two things are contradictory, but as needed to make a choice, it partly reflected the real situation of the crafts under the current semi-industrial conditions. The colour, smell and texture of the bamboo disappeared over time. After three years, with the year-round rice harvest and the growth of ducklings, the shelter naturally returned to the environment.

\subsubsection{The bamboo bridge truss, by the University of Hong Kong}

In 2016, Dr John Shao from the University of Hong Kong researched to investigate the possibilities of Moso bamboo (Chinese species) for large span bridges in Chongqing, China. Dr Shao invited the Colombian architect Xavier Pino for six months to build the initial experiments based on his 20 years of experience utilising bamboo. The research aimed to identify the differences of Moso bamboo compared to Guadua bamboo, which is the species used for large span bridges in Colombia. Jorg Stamm and Simon Velez have reported the largest bamboo bridges in Colombia, achieving spans of 50 meters. Is important to mention that these bamboo bridges based their design in the wooden bridges built in Switzerland during the XVIII century by the Grubenmanns brothers, which achieved sigle-spans of 67 meters (Navone, 2003). Due to the lack of structural calculations during that time, the Grubenmanns brothers tested such a structure using wooden scale models and standing over them. The technology 
transfer from a medieval wood bridge to a bamboo bridge was not a big step due to the same logic applied. According to Stamm and Velez, the best way to transfer the technology is through practice, not theory.

As the starting point, Architect Pino assumed that Guadua had more compressive capacity than Moso, but less flexural tolerance. Architect Pino directed the construction experiment by building a 20 meters truss composed by several Moso bamboos arches referenced by CAD coordinates on a steel scaffolding Cartesian plane in the ground, (Figure 2). Bamboo is hollow and light-weight material. Once the truss built, it was erect using a workforce of 10 men with no crane. Due to the flexural tolerance of Moso, it was relatively easy to bend it and place it into the designed position. Finally, the 20 meters bamboo truss obtained precise measures. A Moso bamboo truss utilised more bamboo poles than what a Guadua bamboo truss would use because Moso has a thinner profile section than Guadua. In the end, the experiment demonstrated that utilising the Cartesian plane technique, the indeterminacy of bamboo material can be overcome. The mechanical performance of the Moso bamboo truss had never seen before in the region.
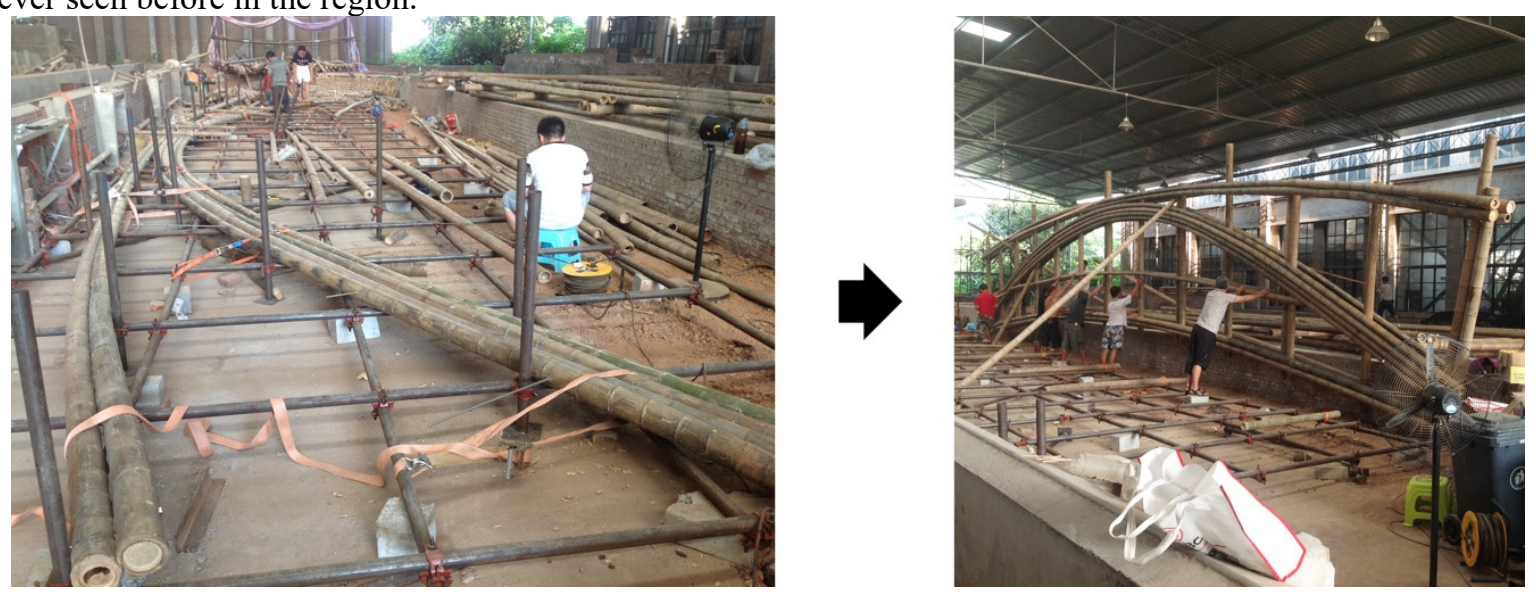

Figure 2. Bamboo arches bent using steel scaffolding as the Cartesian system for reference. The truss was erected mannually by workers. Photographs: Daniel Stamatis.

\subsubsection{Findings across cases}

The cocoon duck shelter utilised mainly split bamboo. Meanwhile the bamboo bridge used full bamboo culms. Both cases used the bending property of bamboo as the main characteristic. The duck shelter was designed intentionally to do not depend on the accuracy and allow error due to the lack of bamboo work experience of students. Meanwhile, the bamboo bridge overcame the indeterminacy of bamboo using the Cartesian plane method to obtain precise measures in every arch.

Moreover, the essence of the cocoon was to return fast to the environment, but the bridge aimed to use preservation treatment to protect bamboo from insects and fungus and, therefore, to assure the long-life performance of its mechanical properties. Furthermore, the cocoon case showed a keen artistic sense, and the bamboo bridge showed an outstanding mechanical performance of full bamboo culm. Further chapters will illustrate how the wing pavilion mixed the Western functionality with an Asian essence by using both cases' techniques in Guangzhou.

1.1.4 The call-to-action from the Nan Sha Bird Park

The Nan Sha Bird Park is a new ecological park in Guangzhou, China, featuring in aquatic birds breeding, science popularisation, and tourism. Thrilled by the potential of bamboo architecture for tourism, the park has been providing real experiences to young architects on what it takes to build with bamboo. In September 2018, students from the school of Architecture and Urban planning at the Huazhong University of Science and Technology were invited to participate in the second bamboo architecture festival to design and build a $16 \mathrm{~m}^{2}$ bamboo structure inside the park. The wing pavilion is the result of this participation.

\section{Research method: participatory action approach}

The particiatory action research approach framed the wing pavilion project. Due to the lack of experience of students on building with bamboo, the participatory action approach was ideal because it leads to proposing new solutions when dealing with problems such as the indeterminacy of bamboo materials, or transferring the digital project into craftsmanship and vice-versa. The participatory action approach creates knowledge through the process of change by focusing on the development and implementation of an action plan. The purpose of this project was to identify the learnability of bamboo construction among un-experienced actors and generalise the variables involved during the creation process of bamboo architecture. The interaction between the students and the Nan Sha Bird Park allowed the problem-solving process to result in a design that gradually gained concreteness until its materialised construction. Initially, students were introduced to bamboo as a building material through a 
series of lectures to provided the more relevant features on the topic in China and abroad. Also, several cases were examined to exemplify the possibilities and to avoid any possible repetition of results. Four HUST tutors assessed six conceptual ideas generated by the students. The "wing" was the selected idea due to its aesthetic value by using the bending property of bamboo, which is one of its main characteristics. The pavilion was designed in Wuhan in 45 days and built in Guangzhou in six days.

The design followed a five-step process: pre-design, formulation, successive answers, project and conclusion of the theory. Handcrafted scale models were created to determine three main aspects of the building: 1) the combination of natural light and artificial light, 2) the unity between structure and furniture, and 3) the complementary materials, such as steel and PVC. McNeel's Rhinoceros ${ }^{\circledR}$ software environment was utilised to translate the information obtained from the handcrafted scale models into digital data. The design resulted in four peers of bamboo arches crossing each other to create a space-roof spanning six meters in total. The unity of the four peers of arches informed the shape of the roof. The technical solution for each arch consisted of two-layers split bamboo attached to one slender bamboo culm bent by heat application technique. Bamboo is a non-standard natural material. A Cartesian plane was utilised as measure reference to ensure that the arch's proportions in the design were the same than the construction. The light-weight arches were pre-fabricated in the park's workshop and carried to the site one kilometre away. Finally, a group of domestic and international bamboo experts evaluated the project. (Figure 3).
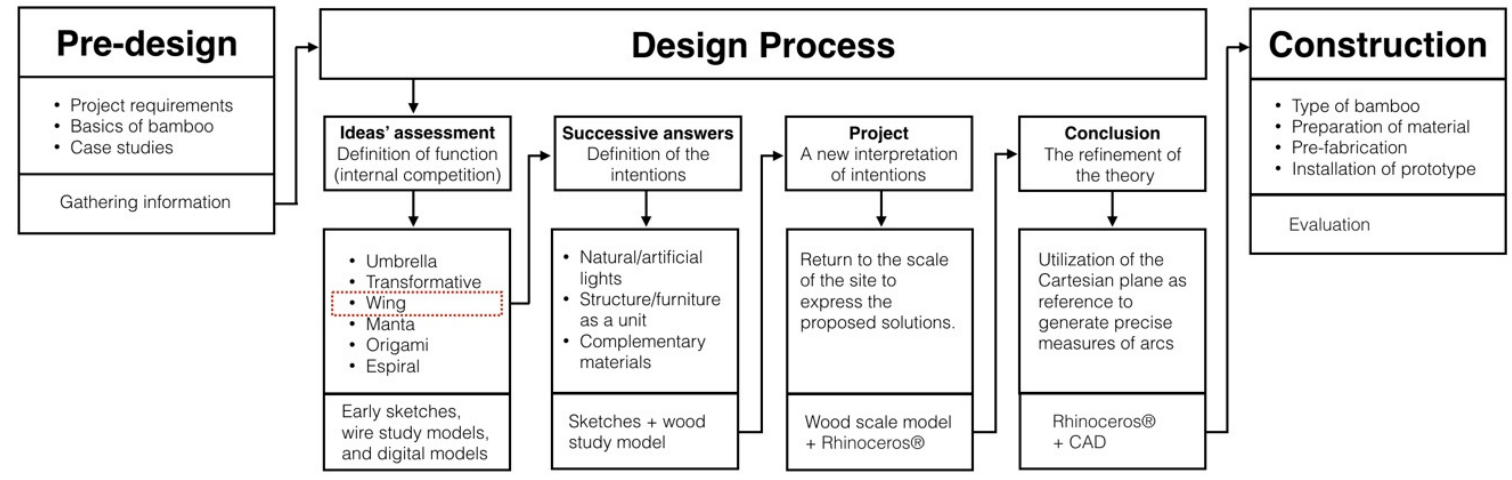

Figure 3. Project's sequence. Image: Daniel Stamatis.

\section{Case description}

The wing pavilion is a $16 \mathrm{~m}^{2}$ temporary, outdoor, public facility designed during September and October, and completed in November 2018, (Figure 4). It is a crossed-arches bamboo structure spanning six meters, capable of sitting four people under a roof coated by a PVC membrane. It performs among the botanical garden and exotic birds. Its joinery is a combination of lashing and iron. The base-foundation consisted of an octagonal metallic base covered by more bamboo. Its design worked through a constant dialogue between traditional craftsmanship and digital tools until sufficient information was gathered to initiate its construction. 


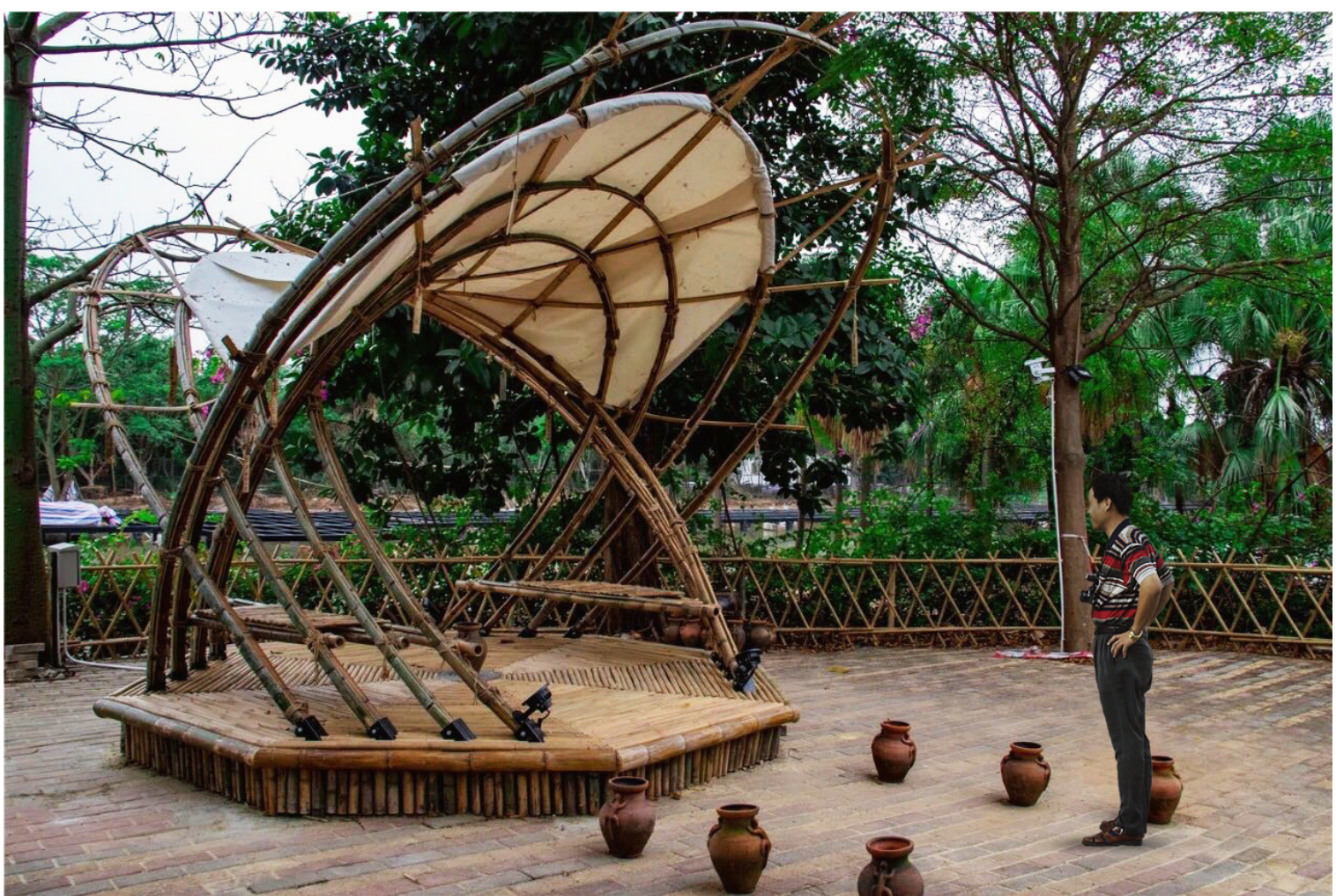

Figure 4. Built prototype of the Wing pavilion during the preesntation day. Photograph: Tan Gangyi.

\subsection{Pre-design phase}

The main objective of the pre-design phase was to discover the project's requirements and determined an understanding of the scope of the project. It reached a critical analysis to be used as a tool during the whole process. The size of the construction area was within a limitation of $4 \times 4 \times 4.2$ meters. At least $1 / 3$ of the structure should hang, most likely to surpass the 1.5 meters. The roof should cover $3 / 4$ of the area and should have at least $1 / 2$ of a rainproof area, (Figure 5). The students decided the function which referred to the human body criteria: stable, secure, and no harsh. The main body structure should be bamboo, but enclosure the possibility to combine it with other materials. The construction should be a prefabricated assembly to reduce the field operation to the minimum. The foundation design should hide as much as possible. After gathering this information, students moved forward to the design phase.

\section{Size limit}

$4 \times 4 \times 4.2$ meters

Roof: $3 / 4$

Rainproof: 1/2

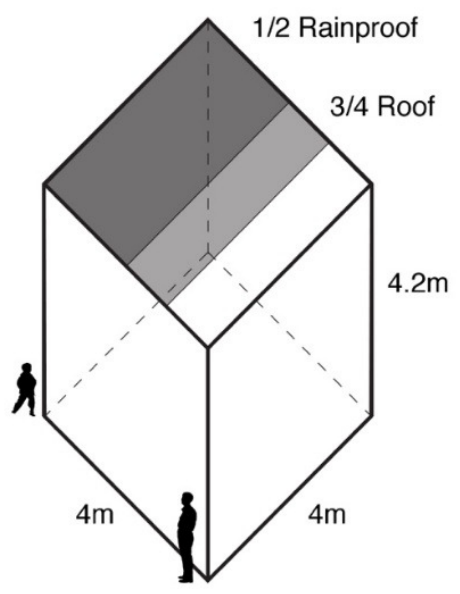

Figure 5. Available area for design implementation. Image: Daniel Stamatis. 


\subsection{Design phase}

This section reports the results of the design phase. After gathering enough information as explained in the predesign section, a series of steps guided the design phase: formulation, successive answers, project, and conclusion of the theory. In many cases, architecture students can get easily overwhelmed by the infinite possibilities of a determined design challenge within a deadline. Controlling the deadline's keystones became a crucial factor to focus on what mattered: the design. During the design phase, several hand-drawn sketches, digital representations, and physical models used a variety of scales.

\subsubsection{Formulation of the function: the subliminal influences}

This step was an attempt to define the interests more than the solutions, and determine the relations and conflicts to be established. This task was not about making a building. Instead, the purpose was to generate ideas using the language of architecture that resulted in a structured building. The students described an alternative of function briefly through schemes (plans, sections, and sketches), and provided a critical conclusion. The students showed the intention of covering the open, public area with crossed bamboo arches that sustained each other informing the shape of the roof. With a poetic sense, students generated a series of free-hand drawings looking for results closer to nature, (Figure 6). The name of the pavilion emerged: "the wing pavilion". According to Chinese architect, Wang Shu, the memories associated with nature are reawaken through real personal experience, and it can be reflected only by the freehand drawing. Also, freehand drawings tend to produce more unadorned buildings. It is an active restriction that prevents over-showy spaces and the loss of scale that usually result from early computer utilisation (Wang, 2012).
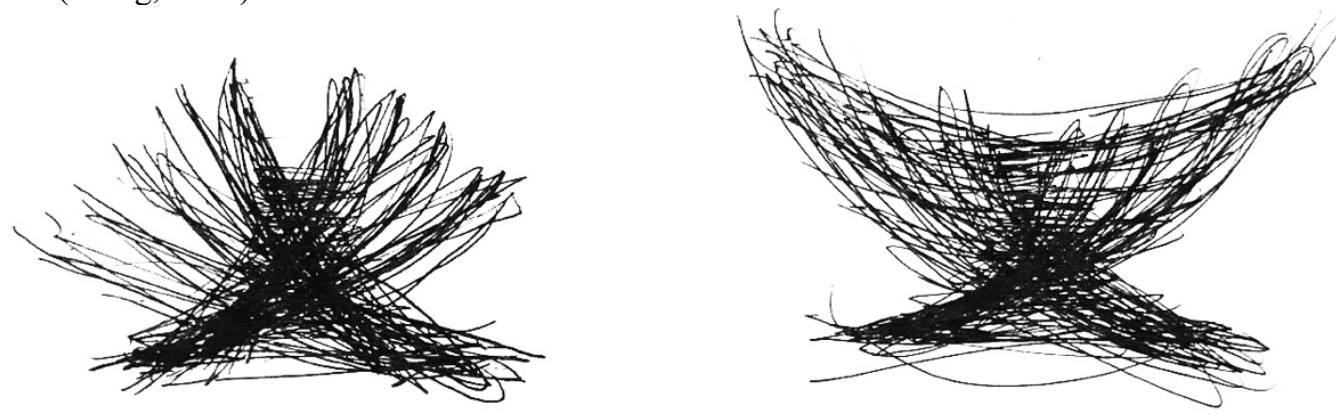

Figure 6. Free-hand sketches defining the interests. Created by Gong Zhichen and Wei Zijie 3.2.2 Successive answers about light, furniture and materials

Here the goal was to manipulate the decision making by breaking the approximation to the project into three essential elements: 1) light, 2) furniture, and 3) materials. A series of exercises were conducted to inform further design decisions.

\subsubsection{Natural vs. artificial light}

A given object can reflect and transmit the light wave. The wing pavilion tested the reflections and transmissions of light by crafting a scale model to answer the following questions: how does light enter into the project?; how could light be altered in space and time?; what about shades and shadows produced by the light and how do they influence a space?; which devices are appropriate to the building and the context?; what is the relationship between artificial lighting and natural lighting in the project?, (Figure 7).
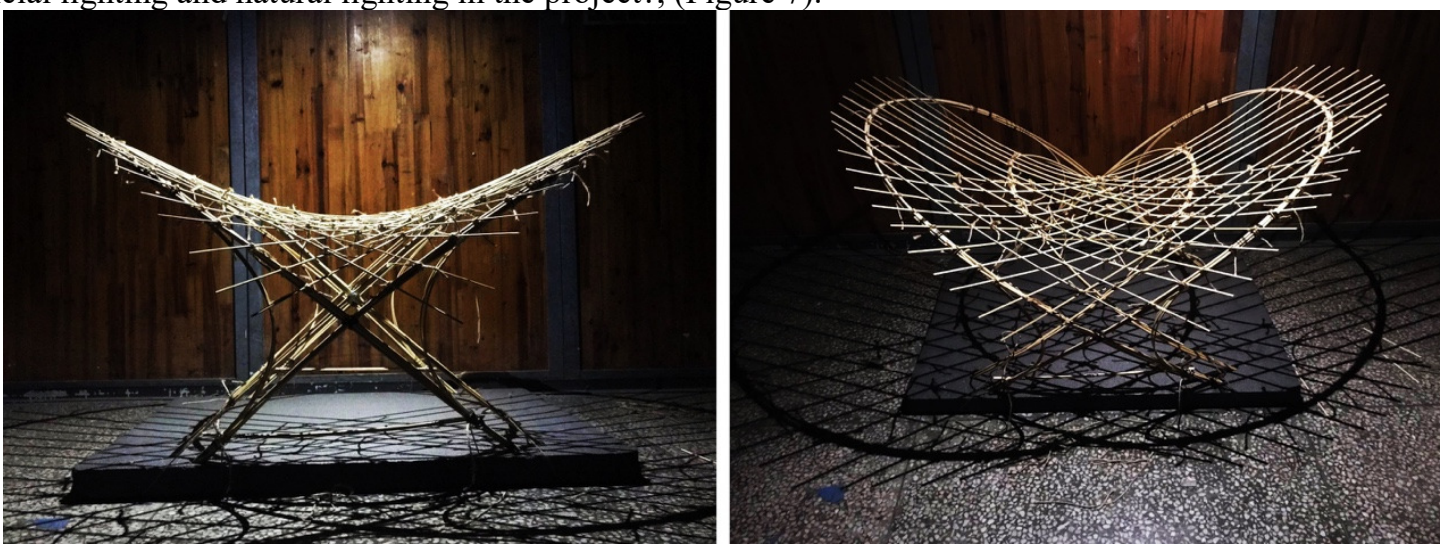

Figure 7. Initial scale model contrasting light effects. Created by Gong Zhichen and Wei Zijie

As bamboo is a natural material, the wing pavilion expected the humidity to accelerate the process of change in the reflection and transmission of light once installed on-site: the green bamboo shall become warm yellow, and the yellow shall become the spotted brown. Moreover, the wing pavilion conceived an indoor/outdoor space. At 
least half of its surface should be rain-proof. Transparency in the roof was intentional. During the day, the shelter shall filter light to the interior through a series of the rhombus that changes as the sunset. During the night, the artificial lighting would be directed to the opposite direction of the structure to provoke contrasts and a cozy environment.

\subsubsection{Furniture and structure merged as a unit}

This exercise went more in-depth into the practice of ergonomics before completed the design. The arches crossing each other suggested a lower element that would reinforce the structure as a whole. Such a feature performed the public seat. As a result, the structure also acted as furniture, with no distinction between them.

\subsubsection{Complementary materials}

To create the arches, a full bamboo culm would break before bending as the design demanded. The wing pavilion required bending split bamboo. By bending bamboo, its outer layer retains the tensile stress, while the softer inner layer undergoes large compressive deformations. The original design proposed to use lashing for the connection between the arches. However, advisors suggested reinforcing with iron rods. The iron may not reflect the characteristics of bamboo, but the low-efficiency of lashing node alone was contrary to the constructive rationality. These two things are contradictory, but as needed to make a choice, it reflected the contemporary situation of the crafts under the semi-industrial conditions. For the foundation, "I" metallic beams were considered along with PVC fabrics for covering the roof.

\subsubsection{The project: a new interpretation of the intentions}

With the assumptions of the previous exercises, this task aimed to revisit the understanding of the project and return to the scale of the site to express the proposed solutions again. As mentioned before, the wing pavilion required to keep the structure inside a given area of $4 \times 4 \times 4.2$ meters. However, the first study model resulted in $25 \%$ larger. Therefore, the design determined that rotating the whole structure to $45^{\circ}$ would be useful to cover the most significant area but within limits, (Figure 8).
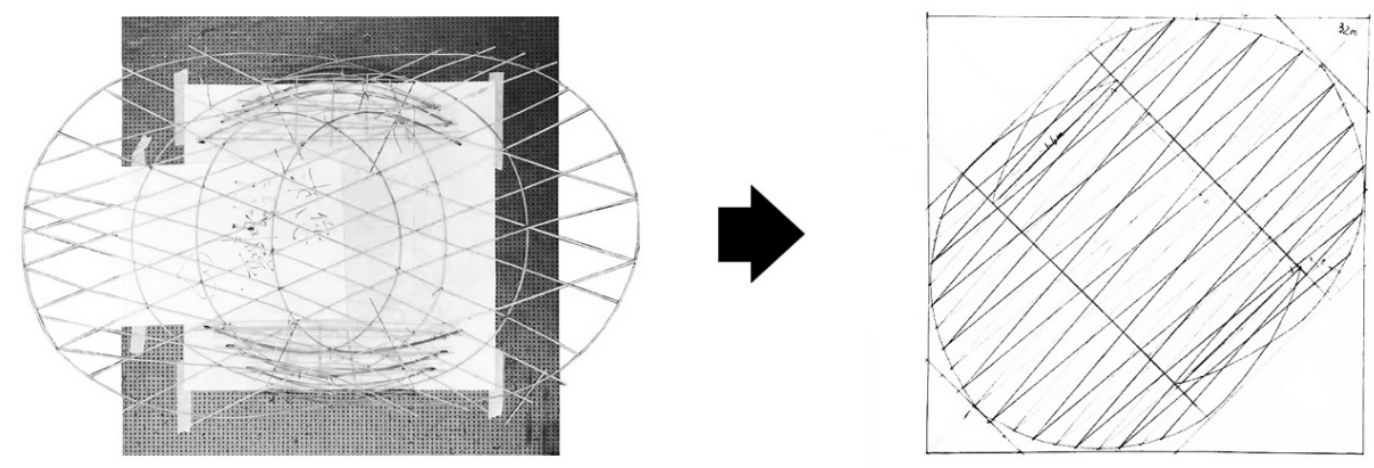

Figure 8. Adjustment of the intentions into the real scale inside the limits of $4 \times 4$ meters. Created by Gong Zhichen and Wei Zijie

Once the scale adjusted to the real area, students developed a digital model using McNeel's Rhinoceros ${ }^{\circledR}$ software environment. Since the site had a brick floor, the design decision-making for the foundation defined a cross-shaped iron base that would separate the bamboo from the ground, protecting it from possible humidity absorption, (Figure 9a). Also, such a metallic base would have attached to it 16 metallic holes to receive the prefabricated bamboo arches, (Figure 9b). Finally, the digital model represented the seats, the roof rhombus grid, and the coverage of bamboo over the metallic base, provoking an octagonal-shaped base at the end, (Figure 9c).
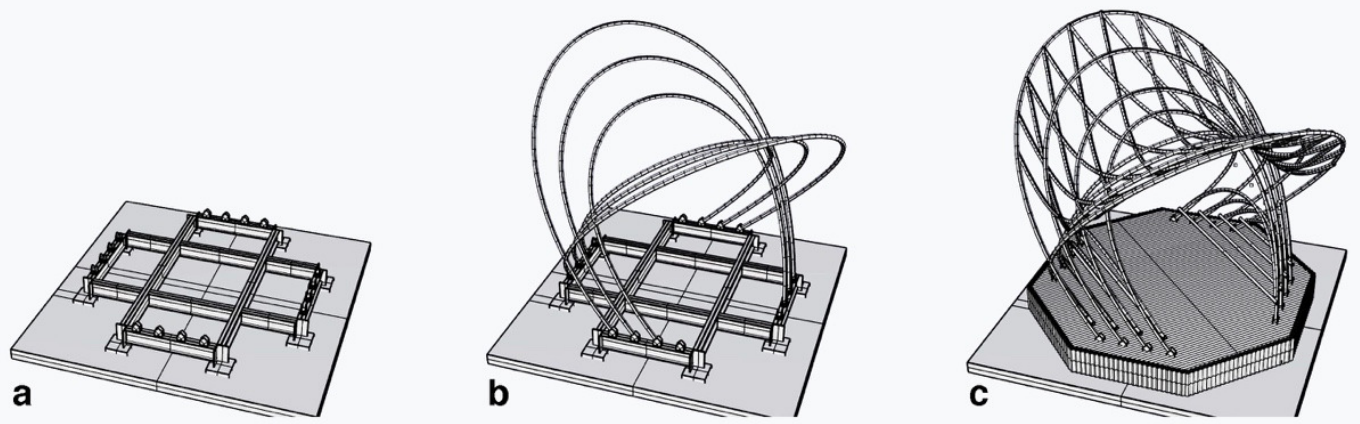

Figure 9. McNeel's Rhinoceros modeling of the Wing pavilion. Created by Gong Zhichen and Wei Zijie 3.2.4 The conclusion of the theory

Using McNeel's Rinoceros ${ }^{\circledR}$ digital model, students generated precise measures for each one of the eight arches. This process clarified the construction planning: a Cartesian plane drawn in the ground as a guide for the curvature of each arch, individually, (Figure 10). After pre-fabricating the light-weight arches, it would be relatively easy to 
erect them and place them in their designed position. The Cartesian plane technique empowers small communities or private business with limited resources to build light-weight bamboo structures. Its main advantage is the facilitation of producing accurate measures to overcome the indeterminacy of bamboo material. Despite the lack of experience on bamboo construction of students, the Cartesian plane minimised errors during the design-toconstruction process.

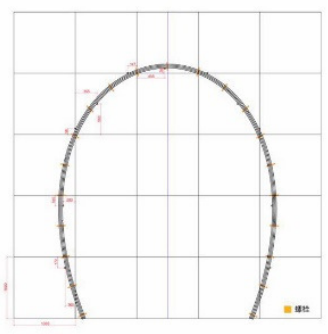

Arch 1

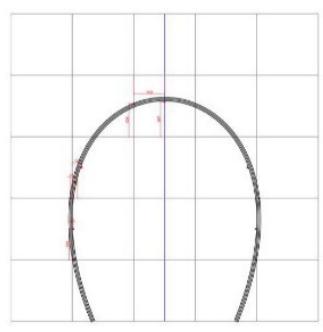

Arch 2

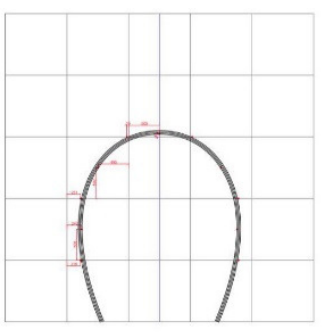

Arch 3

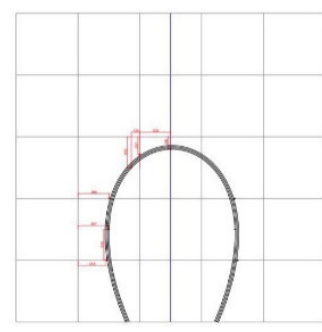

Arch 4

Figure 10. Arches referenced in Cartesian plane, grid 5x5 meters. Created by Gong Zhichen and Wei Zijie

\subsection{Construction}

\subsubsection{The bamboo type}

There are more than 1,600 species of bamboo. The wing pavilion used the Moso bamboo (Phyllostachys edulis) because it is the more available and productive bamboo species in China. However, Moso bamboo does not grow naturally in the region of Guangzhou. The organisers, therefore, purchased it from the central areas of Mainland China. The Moso bamboo utilised was un-treated for preservation, making it less resistant against fungus and insects. It was expected a 5-years life-cycle before returning to the environment.

\subsubsection{Preparation of the material}

The design required 140 meters of split bamboo, $4 \mathrm{~cm}$ width, and 60 meters of slender bamboo culms $\varnothing 5-3 \mathrm{~cm}$. Bamboo poles were washed by hand in a concrete pool for five hours. Then, those were taken to the local factory to split them. The machine utilised was a specialised semi-automatic machine, commonly found in industrial bamboo factories in China.

\subsubsection{Pre-fabrication}

The combination of the fibre-rich outer part and the compressible inner part are responsible for the flexural ductility of split bamboo. Therefore, it is technically easy to bend split bamboo manually. After a first test, students identified that the split bamboo arch itself collapsed for a span of 4 meters. Therefore, a slender bamboo culm was attached to it as a reinforcement, using iron rods and lashing. Students manipulated the bamboo curvature into the Cartesian plane's reference by heat application. Applying heat to bend bamboo is a common practice in China, but less known in South American countries. Although the mechanical properties of bamboo are affected due to the carbonisation process provoked by fire, it can still perform for low loads, reinforcements, furniture, and decorative elements. The arches achieved reliable stability. Such a process was repeated eight times, (Figure 11).
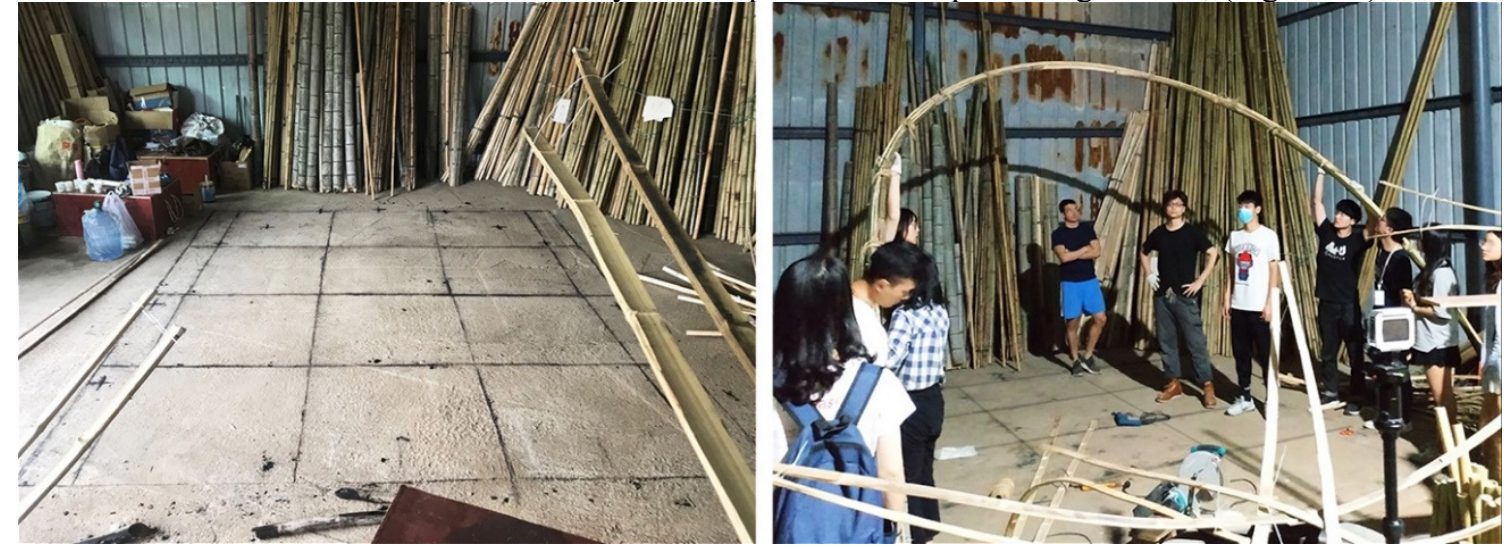

Figure 11. First triumphant bamboo arch using the Cartesian system for referencing measures from the computer design. Photographs: Chen Yudie and Li Meishuang.

\subsubsection{Installation}

After the pre-fabrication process, the eight arches were hand-carried to the site, one kilometre away from the workshop. Each arch was installed individually, from big to small. Six days for construction were not enough to complete the full structure. Therefore students worked day and night. The bamboo arches introduced into the metallic base holes and then fixed with iron, (Figure 12). The split bamboos devoted for the rhombus roof were 
not sufficient due to the material used during the failed tests of the arches at the beginning of the pre-fabrication, resulting in a weaker roof than the expected in the design. Finally, a PVC fabric was placed over the central arches as a roof to protect visitors from rainwater and direct sunlight.
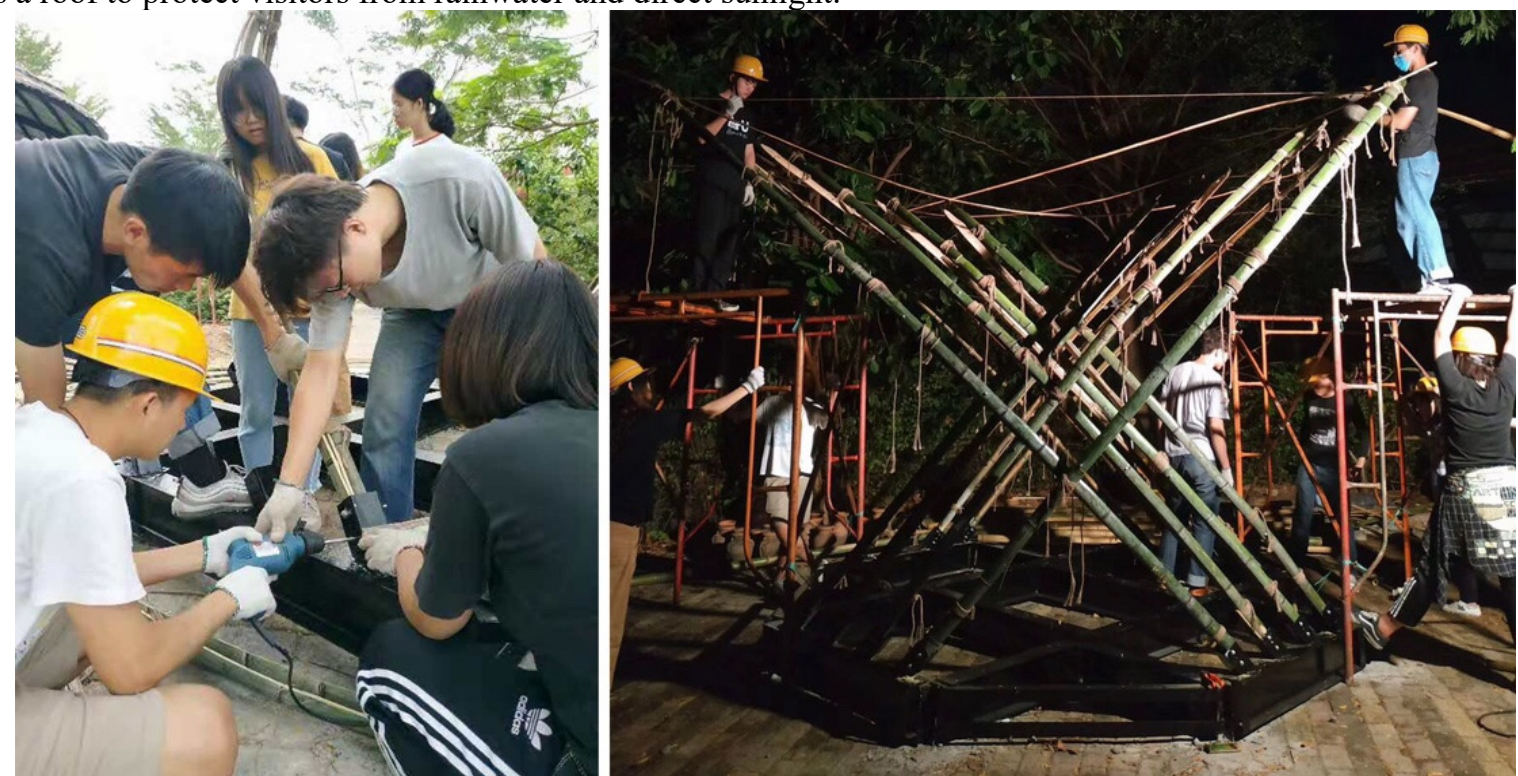

Figure 12. Installation of the eight arches on the metallic base. Photographs: Chen Yudie and Li Meishuang. 3.3.5 Utilisation and evaluation

On December 8, 2018, the wing pavilion, among other bamboo projects, was unveiled officially to the public. More than 80 guests including internationally renowned architects and academicians from Thailand, Indonesia, Malaysia, Singapore, Hong Kong, Macau, Vietnam, United States, Portugal, Belgium, and Italy could use the spot to feel the charm of bamboo and the rigour of the building blended, (Figure 13). The project's evaluation was chaired by the Association of Southeast Asian Designers, who highlighted the outstanding reflection of the flexibility of bamboo and criticised that the roof structure was weaker and its details could be fuller. Finally, the committee added that, unfortunately, the seats were limited to four people. The wing pavilion will remain in the park for at least five years, allowing further visitors to experience the symphony of nature and architecture within a low-carbon and environmentally friendly based pavilion.

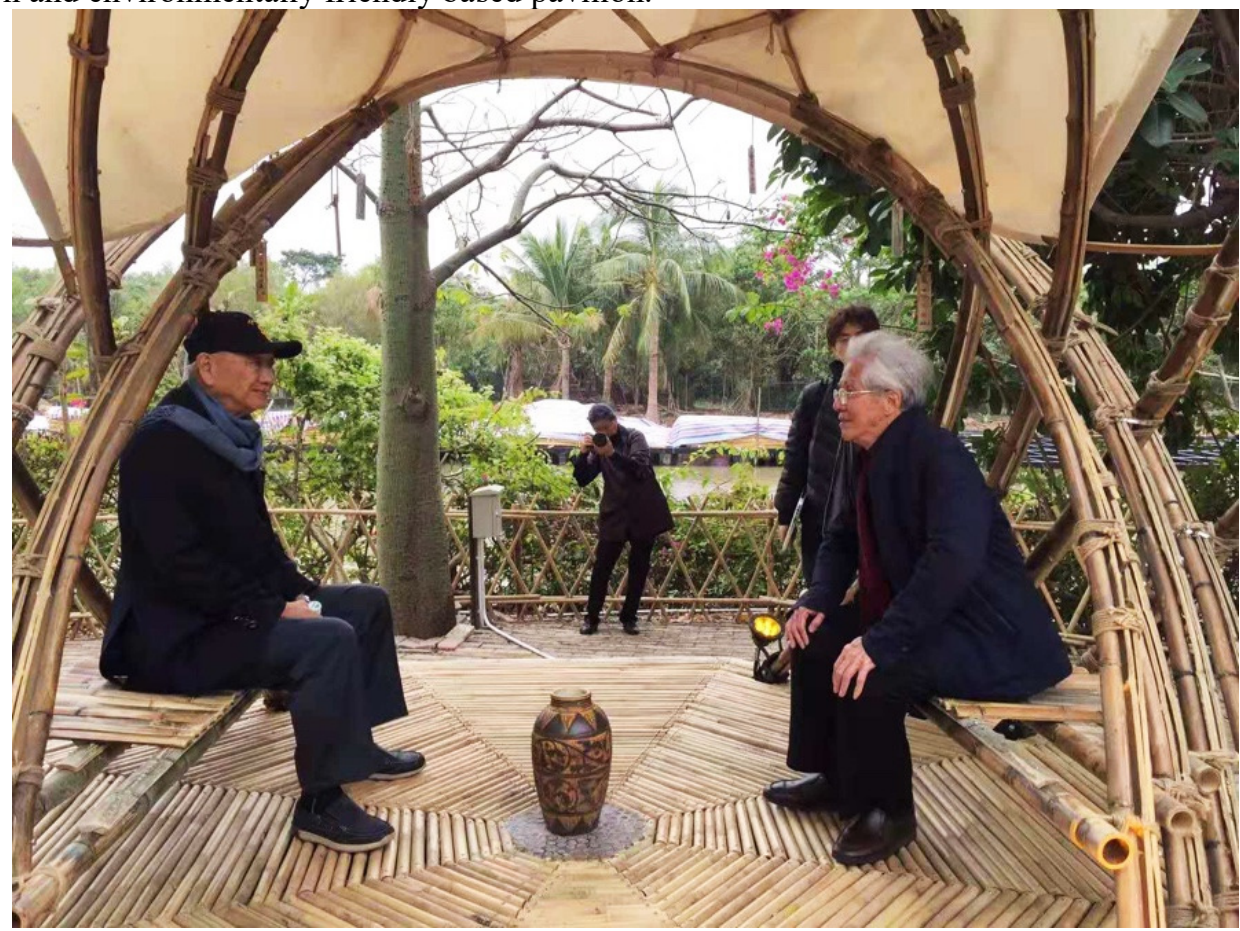

Figure 13. Modernity and tradition, furniture and structure, merged into a unit. Photograph: Tan Gangyi 


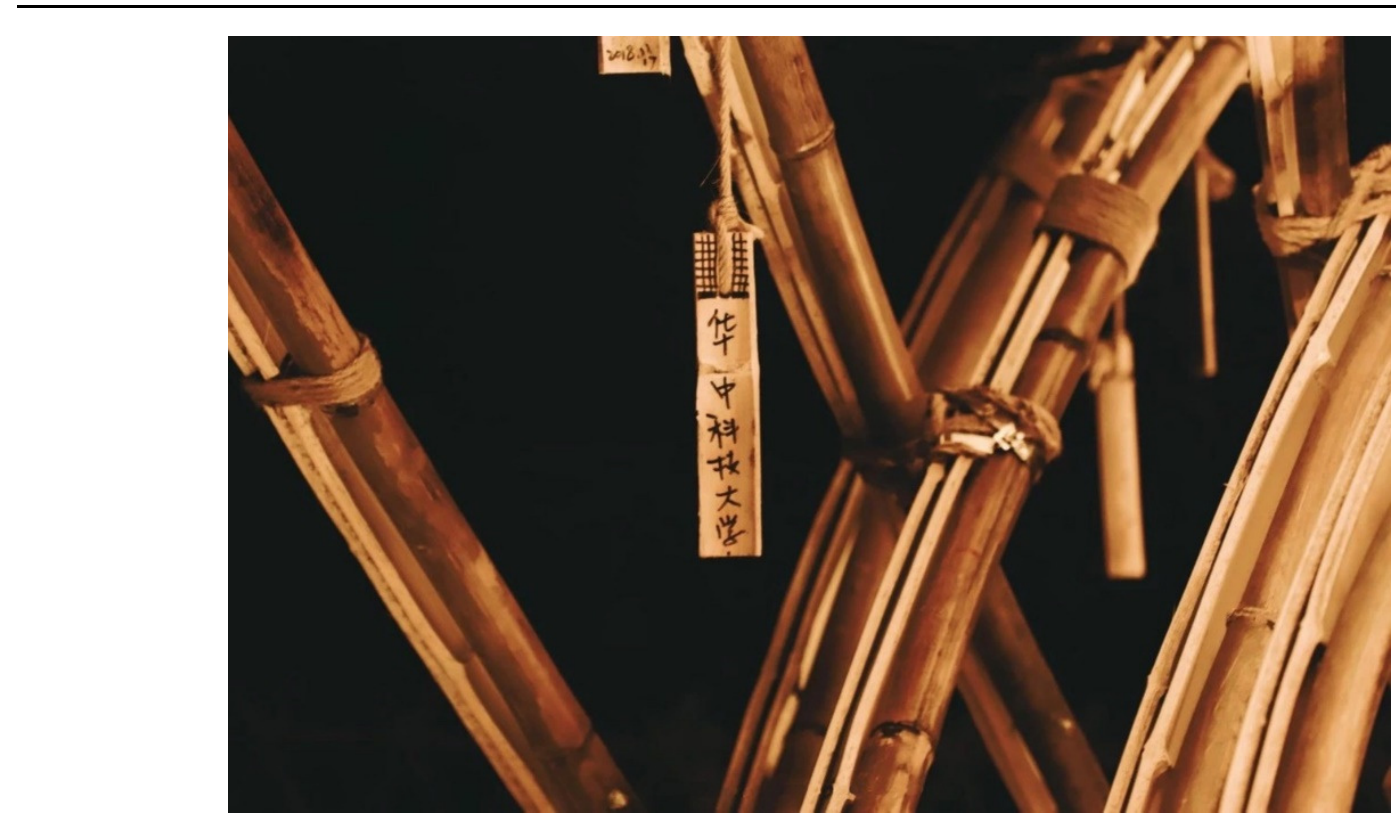

Figure 14. Close-up showing the interconnection between arches, illuminated artificially at night. Photograph: Tan Gangyi

\section{Conclusion}

The Nan Sha Bird Park in collaboration with the School of Architecture and Urban Planning of the Huazhong University of Science and Technology used bamboo because of its strength, beauty, and flexibility, and also because with its 4-year growth cycle and carbon sequestration capacity it is the most environmentally conscientious building material conceivable. Along the design process, the use of natural bamboo texture and material properties stimulated students' thinking and innovation in building a structure within a cooperation environment. The wing pavilion added a touch of beauty to the Nan Sha Bird Park's ecology by providing an attractive public place to enjoy the gift of the natural environment.

The two case studies discussed in the background section (the cocoon duck shelter and the bamboo bridge truss) influenced the design decision-making of the wing pavilion importantly. For instance, the cocoon utilised split bamboo, while the bridge truss utilised full culm. The wing pavilion adopted both, by bending them together as a single element that could not work one without the other. Furthermore, the cocoon duck shelter was designed intentionally to allow error range due to the lack of bamboo work experience of Southeast University students. However, despite the same lack of expertise of HUST students, the wing pavilion learned from the bridge truss about the use of the Cartesian plane to overcome such an indeterminacy of bamboo material. The cocoon showed substantial artistic value. Meanwhile, the bridge truss demonstrated outstanding performance for the mechanical properties of full bamboo culm in large spans. The wing pavilion absorbed both of such qualities by bending the split bamboo to its full potential and reinforcing it with a slender bamboo culm. In general, the wing pavilion mixed the Western functionality with an Asian essence. Nevertheless, the final result presented problems: 1) Poor protection by design: the PVC fabric was installed over the internal two arches, allowing rainwater to fall directly into the seats; and 2), students installed insufficient split bamboo on the roof to reinforce the connection between arches. The short time available and the limited amount of material devoted to the project, which was over-utilised during the failed tests of the first arches, caused both problems. In general, the details could be fuller. More than a perfect result, the wing pavilion aimed to provide the younger generation of architects an experience on what it takes to build with bamboo.

The technique investigated was low, and its learnability was high. The arch, as an architectural element, has been used since ancient times, initially in stone or timber, more recently in reinforced concrete or steel. The bamboo arches of the wing pavilion demonstrated that many of the now underprivileged people could benefit from it since bamboo is an abundant resource and a symbol of the Chinese culture. The combination of computational techniques with traditional handcraft techniques is imminent. The utilization of iron and bamboo reflected the contemporary situation of the crafts under the semi-industrial conditions. The wing pavilion is a prototype that can be improved and replicated in larger scales using the same methodology and construction techniques. Is recommendable to build a preliminary structure scale 1:1 before the definitive construction in further projects. A standard limitation is a budget. The scale modelling was essential for the digital tools, and vice-versa. Bamboo is one of the oldest materials used by humankind to increase comfort and well-being. In today's world of plastics and steel, bamboo continues to make its age-old contributions and is even growing in importance for architectural 
education.

\section{Acknowledgement}

Students of the Innovation and Entrepreneurship Training Program of the School of Architecture and Urban Planning at the Huazhong University of Science and Technology, Wuhan, China: Gong Zhichen, Wei Zijie, Chen Yudie, Li Meishuang, Miao Rui, Chen Yu, Pan Yihan, Deng Yurui, Cheng Li, Gao Mengrong, Li Weicheng.

\section{References}

Botz-Bornstein, Th. (2009). "Wang Shu and the possibilities of architectural regionalism in China", Nordic Journal of Architectural Research. 21(1), 14.

Crolla, K. (2017). "Building indeterminacy modeling - the 'ZCB Bamboo Pavilion' as a case study on nonstandard construction from natural materials." Visualization in Engineering. 5(15), 2-12. https://doi.org/10.1186/s40327-017-0051-4

Flynn, A., Chan, H.Z., Zhu, L. \& Yu, L. (2017). "Sustainability, space and supply chains: the role of bamboo in Anji County, China." Journal of Rural Studies. 49, 128-139. http://dx.doi.org/10.1016/j.jrurstud.2016.11.012

Friederich. H. (2019). Bamboo: Nature's forgotten solution for green industry. <https://www.ecobusiness.com/opinion/bamboo-natures-forgotten-solution-for-green-industry/> (March 2, 2019)

INBAR (2018). "INBAR Annual Report 2018." <https://www.inbar.int/inbars-annual-highlights-2018-isonline/> (May 8, 2019)

Marsden, T., Yu, L. \& Flynn, A. (2011). "Exploring ecological modernization and urban-rural Eco-developments in China: the case of Anji County." The Town Planning Review. 2, 82. https://doi.org/10.3828/tpr.2011.13

McClure, F.A. (1982). Bamboo as a building material. (33 ed.) United States: Foreign Agricultural Service, United States Department of Agriculture. (Chapter 1)

Navone, M. (2003). John Soane and the Wooden Bridges of Switzerland: Architecture and the Culture of Technology from Palladio to Grubenmanns (Cataloghi). United Kingdom: Archivio del Moderno, Acacemia die architettura, Mendriso. (Chapter 3)

Van der Lugt, P. (2017). Booming Bamboo: The (re)discovery of a sustainable material with endless possibilities. The Netherlands: Jeroen van Oostveen. (Chapter 2)

Wang, Sh. (2012). Wang Shu: Imagining the house. Switzerland: Lars Muller Publishers. (Chapter 1)

Xinping, Y., Chenhao, J., Xueying, W., Zilong, L. \& Yucheng, J. (2018). "Research on the applicability of lowtech bamboo architecture in new rural construction." MATEC Web of conferences. 175, 4013. $10.1051 /$ matecconf $/ 201817504013$

Daniel Stamatis. Ph.D candidate of the School of Architecture and Urban Planning at Huazhong University of Science and Technology. His research focuses on a comparison of bamboo architecture in China and abroad. In 2011, he was trained in Colombia by Jorg Stamm in bamboo construction techniques, and in 2012 was certified in bamboo cultivation, processing and utilisation for developing countries by CBRC China National Bamboo Research Center, in Hangzhou.

Wang Zhen. Ph.D of the School of Architecture and Urban Planning at Huazhong University of Science and Technology. His research interests are Green Architecture, Eco-city design, Urban microclimate and urban morphology. He holds a number of national and international awards such as the Teaching Achievement Award of Architectural Design, and has been in charge of several projects including National Science Foundation of China. Liu Hui. Master Degree in Engineering of the School of Architecture and Urban Planning at Huazhong University of Science and Technology. She was the deputy director of the Department of Architecture from 2005 to 2013 and the assistant of the Dean from 2017-present. She undertakes undergraduate and master's courses in architectural design, construction technology, and new energy construction. As a national first-class registered architect, she actively participates in design practice and promotes teaching.

Tan Gangyi. Ph.D, Professor Head of School of Architecture and Urban Planning at the Huazhong University of Science and Technology. His research interests are related to vernacular architecture, the conservation of culture heritage and architectural design. He holds awards such as the First-place Award of Excellence, UNESCO AsiaPacific Heritage Awards for Culture Heritage Conservation, 2003; and the First-place Award of Excellence design in Guangdong, Guangdong Architecture Association, 2006. 